\title{
ON EXISTENCE OF EQUILIBRIUM PAIR FOR CONSTRAINED GENERALIZED GAMES
}

\author{
P. S. SRINIVASAN AND P. VEERAMANI
}

Received 28 August 2003 and in revised form 11 November 2003

We obtain sufficient conditions for the existence of an equilibrium pair for a particular constrained generalized game as an application of a best proximity pair theorem.

\section{Introduction}

Consider the following game involving $n$ players. For the $i$ th player a pair $\left(X_{i}, Y_{i}\right)$ of strategy sets is associated. Knowing the choice of strategies $x^{i} \in X^{i}=\prod_{j=1, j \neq i}^{n} X_{j}$ of all other players, the $i$ th-player choice is restricted to $A_{i}\left(x^{i}\right) \subseteq Y_{i}$. Otherwise the choice will be made from $X_{i}$. According to these preferences, let $f_{i}: Y_{i} \times X^{i} \rightarrow \mathbb{R}$ be the payoff function associated with the $i$ th player for each $i=1, \ldots, n$. In this situation, it is natural to expect an optimal approximate solution which will fulfill the requirement to some extent. Therefore, it should be contemplated to find a pair $(x, y)$ where $x \in X=\prod_{i=1}^{n} X_{i}$ and $y \in Y=\prod_{i=1}^{n} Y_{i}$ which will behave like an equilibrium point of a generalized game, that is, $y_{i} \in A_{i}\left(x^{i}\right)$ and $\max _{z \in A_{i}\left(x^{i}\right)} f_{i}\left(z, x^{i}\right)=f_{i}\left(y_{i}, x^{i}\right)$ for each $i=1, \ldots, n$, and satisfy the optimization constraint, namely, the distance between $x$ and $y$ is minimum with respect to $X$ and $Y$. In this case, the pair $(x, y)$ is called an equilibrium pair and the game is termed as constrained generalized game. Indeed, in this paper, sufficient conditions for the existence of an equilibrium pair for this constrained generalized game are obtained as an application of a best proximity pair theorem.

The entire edifice of game theory expounds with a mathematical search to strike an optimal balance between persons generally having conflicting interests. Each player has to select one from his fixed range of strategies so as to bring the best outcome according to his own preferences.

Following the pioneering work of Debreu [1], the generalized game is one in which the choice of each player is restricted to a subset of strategies determined by the choice of other players. Mathematically, the situation is described as follows.

Let there be $n$ players. Let $X_{1}, \ldots, X_{n}$ be nonempty compact convex sets in a normed linear space $F$. Let $X_{i}$ be the strategy set and let $f_{i}: X=\prod_{i=1}^{n} X_{i} \rightarrow \mathbb{R}$ be the payoff function for the $i$ th player, for each $i=1, \ldots, n$. Given the strategies $x^{i}$ of all other players, the choice 
of the $i$ th player is restricted to the set $A_{i}\left(x^{i}\right) \subseteq X_{i}$. An equilibrium point in a generalized game is an element $x \in X$ such that for each $i=1, \ldots, n, x_{i} \in A_{i}\left(x^{i}\right)$ and

$$
\max _{y \in A_{i}\left(x^{i}\right)} f_{i}\left(y, x^{i}\right)=f_{i}\left(x_{i}, x^{i}\right)=f_{i}(x),
$$

where the following convenient notations are used.

Notation 1.1. Denote

$$
X=\prod_{i=1}^{n} X_{i}, \quad X^{i}=\prod_{\substack{j=1 \\ j \neq i}}^{n} X_{j} .
$$

A point $x$ of $X$ whose $i$ th coordinate is $x_{i}$ and $x^{i} \in X^{i}$ is written as $\left(x_{i}, x^{i}\right)$.

The above definition of the equilibrium point is a natural extension of the Nash equilibrium point introduced by Nash in [6].

Since then a number of generalizations for the existence of an equilibrium point have been given in various directions. For instance, the existence results of equilibria of generalized games were given by Ding and Tan [2], Tan and Yuan [13], Ionescu Tulcea [4], Lassonde [5], and so forth. For a unified treatment on the study of the existence of equilibria of generalized games in various settings, we refer to Yuan [15].

On the other hand, consider the following economic situation. Suppose that goods are manufactured and sold in different locations. Each location can be both a manufacturing as well as a selling unit. It is agreed that the ultimate place where the goods get sold would be determining the payoff for the goods. Let there be $n$ such locations. For each location, two strategies $X_{i}$ and $Y_{i}$ are associated, one to that of manufacturing unit and other to that of selling unit. Knowing the manufacturing strategy $x^{i}$ of all other locations, the choice of selling strategy at the $i$ th location is restricted to $A_{i}\left(x^{i}\right) \subseteq Y_{i}$. Also, let $f_{i}: Y_{i} \times X^{i} \rightarrow \mathbb{R}$ be the payoff associated with the $i$ th location. Moreover, the cost involved in the travel of goods to different places should also be taken into account. In this situation, one cannot expect an equilibrium point as the strategy sets $X_{i}$ and $Y_{i}$ may be quite different. In view of this stand point, it is natural to expect a pair of points $(x, y)$, where $x \in X=\prod_{i=1}^{n} X_{i}$ and $y \in Y=\prod_{i=1}^{n} Y_{i}$, which will fulfill the requirement as in the case of equilibrium point of a generalized game and also minimize the traveling cost where the traveling cost is denoted by $\|x-y\|$. Therefore, it is contemplated to find a pair of points $(x, y)$ where $x \in X$ and $y \in Y$ such that for $i=1, \ldots, n, y_{i} \in A_{i}\left(x^{i}\right)$,

$$
\max _{z \in A_{i}\left(x^{i}\right)} f_{i}\left(z, x^{i}\right)=f_{i}\left(y_{i}, x^{i}\right)
$$

and $\|x-y\|=d(X, Y)$, where

$$
d(X, Y)=\operatorname{Inf}\{\|a-b\|: a \in X, b \in Y\} .
$$

In this case, the pair $(x, y)$ is called an equilibrium pair for this economic situation which is newly termed as constrained generalized game. 
If the sets $Y_{i}$ coincide with $X_{i}$ for $i=1, \ldots, n$, then $Y=X$ and it is easy to see that the equilibrium pair boils down to a single point $x$ which is an equilibrium point for a generalized game in the sense of Debreu [1].

In this paper, an existence of an equilibrium pair for this constrained generalized game is obtained. For this, a best proximity pair theorem exploring the sufficient conditions which ensure the existence of an element $x \in A$ such that

$$
d(x, T x)=d(A, B)
$$

is obtained in Section 3 for the given nonempty subsets $A$ and $B$ of a normed linear space $F$ and a Kakutani multifunction $T: A \rightarrow 2^{B}$. This result is applied to obtain the existence of an equilibrium pair of the constrained game in Section 4. Indeed, an existence theorem for equilibrium point of a generalized game due to Debreu [1] is obtained as a corollary.

\section{Preliminaries}

This section covers the preliminaries and the results that are required in the sequel.

Let $X$ and $Y$ be nonempty sets. A multivalued map or multifunction $T$ from $X$ to $Y$ denoted by $T: X \rightarrow 2^{Y}$ is defined to be a function which assigns to each element of $x \in X$ a nonempty subset $T x$ of $Y$. Fixed points of the multifunction $T: X \rightarrow 2^{X}$ will be the points $x \in X$ such that $x \in T x$.

Let $X$ and $Y$ be any two topological spaces. Let $T: X \rightarrow 2^{Y}$ be a multivalued map. The map $T$ is said to be upper semicontinuous (resp., lower semicontinuous) if $T^{-1}(A):=$ $\{x \in X: T(x) \cap A \neq \varnothing\}$ is closed (resp., open) in $X$ whenever $A$ is a closed (resp., open) subset of $Y$. Also $T$ is said to be continuous if it is both lower semicontinuous and upper semicontinuous.

A multifunction $T: X \rightarrow Y$ is said to have compact values if for each $x \in X, T(x)$ is compact subset of $Y$. Also, $T$ is said to be a compact multifunction if $T(X)$ is a compact subset of $Y$.

It is known that if $T$ is an upper semicontinuous multifunction with compact values, then $T(K)$ is compact whenever $K$ is a compact subset of $X$ if $X$ is Hausdorff.

A multifunction $T: X \rightarrow 2^{Y}$ is said to be a Kakutani multifunction [5] if the following conditions are satisfied:

(1) $T$ is upper semicontinuous;

(2) either $T x$ is a singleton for each $x \in X$ (in which case $Y$ is required to be a Hausdorff topological vector space) or for each $x \in X, T x$ is a nonempty, compact, and convex subset of $Y$ (in which case $Y$ is required to be a convex subset of a Hausdorff topological vector space).

The collection of all Kakutani multifunctions from $X$ to $Y$ is denoted by $\mathscr{K}(X, Y)$.

A multifunction $T: X \rightarrow 2^{Y}$ from a topological space $X$ to another topological space $Y$ is said to be a Kakutani factorizable multifunction if it can be expressed as a composition of finitely many Kakutani multifunctions.

The collection of all Kakutani factorizable multifunctions from $X$ to $Y$ is denoted by $\mathscr{K}_{C}(X, Y)$. 
If $T=T_{1} T_{2} \cdots T_{n}$ is a Kakutani factorizable multifunction, then the functions $T_{1}$, $T_{2}, \ldots, T_{n}$ are known as the factors of $T$. It is a noteworthy fact that $T$ need not be convex valued even though its factors are convex valued.

Let $A$ be any nonempty subset of a normed linear space $X$. Then $P_{A}: X \rightarrow 2^{A}$ defined by

$$
P_{A}(x)=\{a \in A:\|a-x\|=d(x, A)\}
$$

is the set of all best approximations in $A$ to any element $x \in X$.

It is known that if $A$ is compact and convex subset of $X, P_{A}(x)$ is a nonempty compact convex subset of $A$ and the multivalued map $P_{A}$ is upper semicontinuous on $X$.

A single-valued function $f: X \rightarrow \mathbb{R}$ is said to be quasiconcave if the set

$$
\{x \in X: f(x) \geq t\}
$$

is convex for each $t \in \mathbb{R}$.

\section{Best proximity pair theorem}

Consider the fixed point equation $T x=x$ where $T$ is a nonself operator. If this operator equation does not have a solution, then the next attempt is to find an element $x$ in a suitable space such that $x$ is close to $T x$ in some sense. In fact, a classical best approximation theorem, due to Fan [3], states that if $K$ is a nonempty compact convex subset of a Hausdorff locally convex topological vector space $E$ with a continuous seminorm $p$ and $T: K \rightarrow E$ is a single-valued continuous map, then there exists an element $x_{0} \in K$ such that

$$
p\left(x_{0}-T x_{0}\right)=d\left(T x_{0}, K\right) \text {. }
$$

Later, this result has been generalized by Sehgal and Singh $[10,11]$ to the one for continuous multifunctions. It is remarked that they have also proved the following generalization of the result due to Prolla [7].

If $K$ is a nonempty approximately compact convex subset of a normed linear space $X$, $T: K \rightarrow X$ a multivalued continuous map with $T(K)$ relatively compact, and $g: K \rightarrow K$ an affine, continuous, and surjective single-valued map such that $g^{-1}$ sends compact subsets of $K$ onto compact sets, then there exists an element $x_{0}$ in $K$ such that

$$
d\left(g x_{0}, T x_{0}\right)=d\left(T x_{0}, K\right)
$$

In the setting of Hausdorff locally convex topological vector spaces, Vetrivel et al. [14] have established existential theorems that guarantee the existence of a best approximant for continuous Kakutani factorizable multifunctions which unify and generalize the known results on best approximations.

The known example [11] shows that the requirement of continuity assumption of the involved multifunction in Sehgal and Singh's result [11] cannot be relaxed. 
Example 3.1. Let $X=\mathbb{R}^{2}, K=[0,1] \times\{0\}$, and $g=I$, the identity map. Let $T: K \rightarrow 2^{X}$ be defined by

$$
T(a, 0)= \begin{cases}\{(0,1)\} & \text { if } a \neq 0 \\ \text { the line segment joining }(0,1) \text { and }(1,0) & \text { if } a=0\end{cases}
$$

Then $T$ is upper semicontinuous but not lower semicontinuous. Also it is clear that there is no $x \in K$ such that

$$
d(x, T x)=d(T x, K) .
$$

Remark 3.2. In [12], the above known example has not been quoted correctly. Example 1.1 of [12] should be replaced by the above example.

On the other hand, even though a best approximation theorem guarantees the existence of an approximate solution, it is contemplated to find an approximate solution which is optimal. The best proximity pair theorem (see [9]) sheds light in this direction. Indeed a best proximity pair theorem due to Sadiq Basha and Veeramani [8] provides sufficient conditions that ensure the existence of element $x_{0} \in A$ such that $d\left(x_{0}, T x_{0}\right)=$ $d(A, B)$ where the given $T: A \rightarrow 2^{B}$ is a Kakutani factorizable multifunction defined on the suitable subsets $A$ and $B$ of a topological vector space $E$. The pair $\left(x_{0}, T x_{0}\right)$ is called a best proximity pair of $T$. The best proximity pair theorem seeks an approximate solution which is optimal.

The following fixed point theorem, due to Lassonde [5], for Kakutani factorizable multifunctions will be invoked to establish the main result of this section.

THeOREM 3.3 (Lassonde [5]). If S is a nonempty convex subset of a Hausdorfflocally convex topological vector space, then any compact Kakutani factorizable multifunction $T: S \rightarrow 2^{S}$ (i.e., any compact multifunction in the family $\mathscr{K}_{C}(S, S)$ ) has a fixed point.

Let $A$ and $B$ be any two nonempty subsets of a normed linear space. Before stating the principal result of this section, the following notions are recalled:

$$
\begin{gathered}
d(A, B):=\operatorname{Inf}\{d(a, b): a \in A, b \in B\}, \\
\operatorname{Prox}(A, B):=\{(a, b) \in A \times B: d(a, b)=d(A, B)\}, \\
A_{0}:=\{a \in A: d(a, b)=d(A, B) \text { for some } b \in B\}, \\
B_{0}:=\{b \in B: d(a, b)=d(A, B) \text { for some } a \in A\} .
\end{gathered}
$$

If $A=\{x\}$, then $d(A, B)$ is written as $d(x, B)$. Also, if $A=\{x\}$ and $B=\{y\}$, then $d(x, y)$ denotes $d(A, B)$ which is precisely $\|x-y\|$.

The following best proximity pair theorem [8] which will be used to prove the existence of an equilibrium pair is included for the sake of completeness.

Theorem 3.4. Let $A$ and $B$ be nonempty compact convex subsets of a normed linear space $X$ and let $T: A \rightarrow 2^{B}$ be an upper semicontinuous multifunction. Further assume that for each $x$ in $A, T x$ is a nonempty closed convex subset of $B$ and $T\left(A_{0}\right) \subseteq B_{0}$. 
Then there exists an element $x \in A$ such that

$$
d(x, T x)=d(A, B)
$$

Proof. Consider the metric projection map $P_{A}: X \rightarrow 2^{A}$ defined as

$$
P_{A}(x)=\{a \in A:\|a-x\|=d(x, A)\} .
$$

As $A$ is a nonempty compact convex set, $P_{A}(x)$ is a nonempty closed, convex subset of $A$, for each $x$ in $A$. Also it is well known that $P_{A}$ is an upper semicontinuous multivalued map.

Now, it is claimed that $P_{A}(T x) \subseteq A_{0}$, for each $x$ in $A_{0}$.

Let $y \in P_{A}(T x)$. Then $y \in P_{A}(z)$, for some $z \in T x$. This implies that $\|x-y\|=d(z, A)$. But it is given that $T\left(A_{0}\right) \subset B_{0}$. Hence $z \in B_{0}$. But $z \in B_{0}$ implies that there exists $a \in A$ such that $\|a-z\|=d(z, A)$. Now

$$
\|z-y\|=d(z, A) \leq\|z-a\|=d(A, B) .
$$

This implies that $\|z-y\|=d(A, B)$. Hence $y \in A_{0}$. Consequently, $P_{A}(T x) \subseteq A_{0}$, for each $x$ in $A_{0}$.

Since $A$ and $B$ are compact sets, $A_{0} \neq \varnothing$. Also it is easy to prove that $A_{0}$ is compact and convex. Now, for $x$ in $A_{0}, P_{A}(T x)$ need not be a convex set. Here, the fixed point theorem of Lassonde [5] is invoked. Though $P_{A} \circ T$ is not a convex-valued multifunction, $P_{A} \circ T$ : $A_{0} \rightarrow 2^{A_{0}}$ is a Kakutani factorizable multifunction. Hence, by the fixed point theorem of Lassonde, there exists $x \in A_{0}$ such that $x \in P_{A}(T x)$.

Now, $x \in P_{A}(T x)$ implies that $\|x-y\|=d(y, A)$, for some $y \in T x$. Then $T x \subseteq B_{0}$ implies that there exists $a \in A$ such that $\|a-y\|=d(A, B)$. Hence

$$
\|x-y\|=d(y, A) \leq\|y-a\|=d(A, B) .
$$

Therefore $\|x-y\|=d(A, B)$. As $d(x, T x) \leq\|x-y\|=d(A, B)$, hence $d(x, T x)=d(A, B)$. This proves the theorem.

Remark 3.5. In [8], the above theorem is proved in more general setup where the set $A$ is approximately compact and $T$ is a Kakutani factorizable multifunction.

\section{Constrained generalized game}

This section is devoted to principal results on game theory.

The following lemma is an important tool in the proof of Theorem 4.4. For the proof, we refer to [12].

Lemma 4.1. Let $A$ and $B$ be nonempty compact subsets in a normed linear space $F$ and let $f: A \times B \rightarrow \mathbb{R}$ be a continuous function. Given a continuous multifunction $T: A \rightarrow 2^{B}$ with compact values, the function $g: A \rightarrow \mathbb{R}$ defined by $g(x)=\delta(T x, x):=\max _{z \in T(x)} f(z, x)$ is a continuous function.

The proof of the principal theorem of this section invokes the best proximity pair theorem (Theorem 3.4). Before that, the following definitions are introduced. 
Let $X_{1}, \ldots, X_{n}$ and $Y_{1}, \ldots, Y_{n}$ be nonempty compact convex sets in a normed linear space $F$. Also, let $X=\prod_{i=1}^{n} X_{i}, Y=\prod_{i=1}^{n} Y_{i}$, and

$$
X_{0}=\{x \in X:\|x-y\|=d(X, Y) \text { for some } y \in Y\} \text {. }
$$

Definition 4.2. Let $f_{i}: Y_{i} \times X^{i} \rightarrow \mathbb{R}$, for $i=1, \ldots, n$, be $n$ single-valued functions. These $n$ functions are said to satisfy a condition (A) with respect to the given multifunctions $A_{i}: X^{i} \rightarrow 2^{Y_{i}}$ if for each $x \in X_{0}$ and for all $y \in Y$ such that

$$
\begin{gathered}
y_{i} \in A_{i}\left(x^{i}\right), \\
\delta_{i}\left(A_{i}\left(x^{i}\right), x^{i}\right):=\max _{z \in A_{i}\left(x^{i}\right)} f_{i}\left(z, x^{i}\right)=f_{i}\left(y_{i}, x^{i}\right) \quad \text { for each } i=1, \ldots, n,
\end{gathered}
$$

there exists $a \in X$ such that $\|a-y\| \leq d(X, Y)$.

Definition 4.3. Let the single-valued functions $f_{i}: Y_{i} \times X^{i} \rightarrow \mathbb{R}$ and the multifunctions $A_{i}$ : $X^{i} \rightarrow 2^{Y_{i}}$, for $i=1, \ldots, n$, be given. Let $x \in X$ and $y \in Y$ be such that, for each $i=1, \ldots, n$,

(a) $y_{i} \in A_{i}\left(x^{i}\right)$

(b) $\delta_{i}\left(A_{i}\left(x^{i}\right), x^{i}\right):=\max _{z \in A_{i}\left(x^{i}\right)} f_{i}\left(z, x^{i}\right)=f_{i}\left(y_{i}, x^{i}\right)$,

(c) $\|x-y\|=d(X, Y)$.

Then the pair $(x, y)$ is called an equilibrium pair for the game which is termed as constrained generalized game.

Theorem 4.4. Let $X_{1}, \ldots, X_{n}$ and $Y_{1}, \ldots, Y_{n}$ be nonempty compact convex sets in a normed linear space $F$. For $i=1, \ldots, n$, let $f_{i}: Y_{i} \times X^{i} \rightarrow \mathbb{R}$ be continuous functions satisfying $a$ condition (A) with respect to the given lower semicontinuous multifunctions $A_{i}: X^{i} \rightarrow 2^{Y_{i}}$, $i=1, \ldots, n$, in $\mathscr{K}\left(X^{i}, Y_{i}\right)$, and are such that for any fixed $x^{i} \in X_{i}$, the function $y_{i} \rightarrow f_{i}\left(y_{i}, x^{i}\right)$ is quasiconcave on $X_{i}$ for each $i=1, \ldots, n$. Then there exist an equilibrium pair for the constrained generalized game.

Proof. For each $i=1, \ldots, n$, let the multifunction $E_{i}: X^{i} \rightarrow 2^{Y_{i}}$ be defined as follows:

$$
E_{i}\left(x^{i}\right)=\left\{y_{i} \in A_{i}\left(x^{i}\right): f_{i}\left(y_{i}, x^{i}\right)=\delta_{i}\left(A_{i}\left(x^{i}\right), x^{i}\right)\right\}
$$

and $E: X \rightarrow 2^{Y}$ as

$$
E(x)=\prod_{i=1}^{n} E_{i}\left(x^{i}\right) .
$$

It is shown that $E$ satisfies all the conditions of Theorem 3.4. For this, it is claimed that $E_{i} \in \mathscr{K}\left(X^{i}, Y_{i}\right)$, for $i=1, \ldots, n$.

Let $i \in\{1, \ldots, n\}$ be fixed. For any fixed $x^{i} \in X^{i}, E_{i}\left(x^{i}\right)$ is nonempty and compact because the function $y_{i} \rightarrow f_{i}\left(y_{i}, x^{i}\right)$ is continuous on the compact set $A_{i}\left(x^{i}\right)$. Now, it is shown that $E_{i}\left(x^{i}\right)$ is convex.

Let $z_{1}, z_{2} \in E_{i}\left(x^{i}\right)$. This implies

$$
f_{i}\left(z_{1}, x^{i}\right) \geq \delta_{i}\left(A_{i}\left(x^{i}\right), x^{i}\right), \quad f_{i}\left(z_{2}, x^{i}\right) \geq \delta_{i}\left(A_{i}\left(x^{i}\right), x^{i}\right) .
$$


Since $y_{i} \rightarrow f_{i}\left(y_{i}, x^{i}\right)$ is quasi concave on $X_{i}$,

$$
f_{i}\left(\lambda z_{1}+(1-\lambda) z_{2}, x^{i}\right) \geq \delta_{i}\left(A_{i}\left(x^{i}\right), x^{i}\right) .
$$

But, $A_{i}\left(x^{i}\right)$ is a convex set. So,

$$
f_{i}\left(\lambda z_{1}+(1-\lambda) z_{2}, x^{i}\right) \leq \delta_{i}\left(A_{i}\left(x^{i}\right), x^{i}\right) .
$$

Therefore,

$$
f_{i}\left(\lambda z_{1}+(1-\lambda) z_{2}, x^{i}\right)=\delta_{i}\left(A_{i}\left(x^{i}\right), x^{i}\right) .
$$

Hence $\lambda z_{1}+(1-\lambda) z_{2} \in E_{i}\left(x^{i}\right)$. Therefore, $E_{i}\left(x^{i}\right)$ is convex for each $i=1, \ldots, n$.

Next, it is shown that $E_{i}: X^{i} \rightarrow 2^{Y_{i}}$ is upper semicontinuous multifunction on $X_{i}$, for every $i=1, \ldots, n$.

Let $z_{n} \in X^{i}$ with $z_{n} \rightarrow z$ and $w_{n} \in E_{i}\left(z_{n}\right)$ with $w_{n} \rightarrow w$.

The fact $w_{n} \in E_{i}\left(z_{n}\right)$ implies the fact that $f_{i}\left(w_{n}, z_{n}\right)=\delta_{i}\left(A_{i}\left(z_{n}\right), z_{n}\right)$. By Lemma 4.1, $x^{i} \rightarrow \delta_{i}\left(A_{i}\left(x^{i}\right), x^{i}\right)$ is a continuous function. Therefore, $\delta_{i}\left(A_{i}\left(z_{n}\right), z_{n}\right) \rightarrow \delta_{i}\left(A_{i}(z), z\right)$. Moreover, since $f_{i}$ is a continuous function, $f_{i}\left(w_{n}, z_{n}\right) \rightarrow f_{i}(w, z)$. This implies that $f_{i}(w, z)=$ $\delta_{i}\left(A_{i}(z), z\right)$. Hence $w \in E_{i}(z)$. Therefore $E_{i}$ is upper semicontinuous on $X_{i}$ for every $i=$ $1, \ldots, n$. Hence this establishes the claim that $E_{i} \in \mathscr{K}\left(X^{i}, Y_{i}\right)$, for $i=1, \ldots, n$. Further from the above claim, it follows that $E \in \mathscr{K}(X, Y)$.

Now, let $x \in X_{0}$ and $y \in E(x)$. This implies that $f_{i}\left(y_{i}, x^{i}\right)=\delta\left(A_{i}\left(x^{i}\right), x^{i}\right), i=1, \ldots, n$. Since $f_{i}$ for $i=1, \ldots, n$ satisfy condition (A) with respect to the multifunctions $A_{i}$, there exists $a \in X$ such that $\|a-y\|=d(X, Y)$. This illustrates the fact $y \in Y_{0}$. Therefore $E\left(X_{0}\right)$ $\subseteq Y_{0}$. Hence $E$ satisfies all the conditions of Theorem 3.4. Therefore, there exists $x \in X$ such that

$$
d(x, E x)=d(X, Y)
$$

Since Ex is compact, there exists $y \in E x$ such that

$$
d(x, y)=d(X, Y) .
$$

This establishes the theorem.

If the sets $Y_{i}$ 's coincides with $X_{i}$ 's for $i=1, \ldots, n$, then $Y=X$ and the following corollary is immediate.

Corollary 4.5. Let $X_{1}, \ldots, X_{n}$ be nonempty compact convex sets in a normed linear space $F$. Let $A_{i}: X^{i} \rightarrow 2^{X_{i}}, i=1, \ldots, n$, be lower semicontinuous multifunctions in $\mathscr{K}\left(X^{i}, X_{i}\right)$. For $i=$ $1, \ldots, n$, let $f_{i}: X \rightarrow \mathbb{R}$ be continuous functions such that, for any fixed $x^{i} \in X_{i}$, the function $y_{i} \rightarrow f_{i}\left(y_{i}, x^{i}\right)$ is quasiconcave on $X_{i}$ for each $i=1, \ldots, n$. Then there exists an equilibrium point for the game in the sense of Debreu [1].

Remark 4.6. It is remarked that Theorem 4.4 does not strictly generalize Debreu's theorem [1] or [5, Theorem 6]. In [5] the sets $X_{i}$ 's are convex sets with all the multifunctions $A_{i}$ 's compact except possibly one in addition to the conditions for $A_{i}$ 's given in the above corollary. 


\section{References}

[1] G. Debreu, A social equilibrium existence theorem, Proc. Nat. Acad. Sci. USA 38 (1952), 886893.

[2] X. P. Ding and K.-K. Tan, On equilibria of noncompact generalized games, J. Math. Anal. Appl. 177 (1993), no. 1, 226-238.

[3] K. Fan, Extensions of two fixed point theorems of F. E. Browder, Math. Z. 112 (1969), 234-240.

[4] C. Ionescu Tulcea, On the approximation of upper semi-continuous correspondences and the equilibriums of generalized games, J. Math. Anal. Appl. 136 (1988), no. 1, 267-289.

[5] M. Lassonde, Fixed points for Kakutani factorizable multifunctions, J. Math. Anal. Appl. 152 (1990), no. 1, 46-60.

[6] J. F. Nash Jr., Equilibrium points in n-person games, Proc. Nat. Acad. Sci. U.S.A. 36 (1950), 4849.

[7] J. B. Prolla, Fixed-point theorems for set-valued mappings and existence of best approximants, Numer. Funct. Anal. Optim. 5 (1982/1983), no. 4, 449-455.

[8] S. Sadiq Basha and P. Veeramani, Best proximity pairs and best approximations, Acta Sci. Math. (Szeged) 63 (1997), no. 1-2, 289-300.

[9] Best proximity pair theorems for multifunctions with open fibres, J. Approx. Theory 103 (2000), no. 1, 119-129.

[10] V. M. Sehgal and S. P. Singh, On random approximations and a random fixed point theorem for set valued mappings, Proc. Amer. Math. Soc. 95 (1985), no. 1, 91-94.

[11] A generalization to multifunctions of Fan's best approximation theorem, Proc. Amer. Math. Soc. 102 (1988), no. 3, 534-537.

[12] P. S. Srinivasan and P. Veeramani, On best proximity pair theorems and fixed-point theorems, Abstr. Appl. Anal. 2003 (2003), no. 1, 33-47.

[13] K.-K. Tan and X.-Z. Yuan, Approximation method and equilibria of abstract economies, Proc. Amer. Math. Soc. 122 (1994), no. 2, 503-510.

[14] V. Vetrivel, P. Veeramani, and P. Bhattacharyya, Some extensions of Fan's best approximation theorem, Numer. Funct. Anal. Optim. 13 (1992), no. 3-4, 397-402.

[15] G. X.-Z. Yuan, The study of minimax inequalities and applications to economies and variational inequalities, Mem. Amer. Math. Soc. 132 (1998), no. 625, x+140.

P. S. Srinivasan: Department of Mathematics, Indian Institute of Technology, Madras, Chennai 600 036, India

Current address: Department of Mathematics and Statistics, University of Hyderabad, Hyderabad 500 046, India

E-mail address: psssm@uohyd.ernet.in

P. Veeramani: Department of Mathematics, Indian Institute of Technology, Madras, Chennai 600 036, India

E-mail address: pvmani@iitm.ac.in 